\title{
ESTIMACIÓN DE LA FIJACIÓN DE NITRÓGENO EN UNA VEGA DE TIERRA DEL FUEGO POR EL MÉTODO DE LA DIFERENCIA DE NITRÓGENO
}

\author{
ESTIMATION OF NITROGEN FIXATION IN A MEADOW IN TIERRA \\ DEL FUEGO USING THE NITROGEN DIFFERENCE METHOD
}

Sergio Radic ${ }^{1} \&$ James McAdam ${ }^{2}$

\begin{abstract}
Sheep production in Tierra del Fuego is based on extensive grazing of largely native pastures. Production systems focus on meeting the nutritional demands of all classes of sheep from these pastures throughout the year, but there are deficit periods when production is limited by pasture quality and availability. Legumes are the cheapest sources of protein for animals on these grasslands and could be a key component of production systems. There are no published measurements of nitrogen fixation from this region of southern Patagonia. In a field experiment on a meadow located on dryland steppe (300-350 mm year ${ }^{-1}$ ) in Tierra del Fuego (53 $09^{\circ}$ 'south; $68^{\circ} 44^{\prime}$ 'west), grazing exclusion cages were used in established reseeds (on wet and dry areas) to measure the yield and nitrogen fixation of Trifolium repens during the growing season. The nitrogen difference method was used to measure fixation and the experimental design was randomized block analysis with three replicates. The model included area as sources of variation, analysed by analysis of variance. The mean level of nitrogen fixation was $98.7 \mathrm{~kg} \mathrm{~N}^{-1}$ and significantly more $(\mathrm{P} \leq 0.05)$ nitrogen was fixed on wet areas $\left(126 \mathrm{~kg} \mathrm{~N} \mathrm{ha}^{-1}\right)$ than dry areas $\left(71 \mathrm{~kg} \mathrm{~N} \mathrm{ha}^{-1}\right)$.
\end{abstract}

Key word: Trifolium repens, grasslands, Patagonia, soil moisture.

\section{RESUMEN}

La producción ovina en Tierra del Fuego es de carácter extensivo y se basa en el pastoreo directo. En este sentido, los parámetros productivos se encuentran asociados a la satisfacción de los requerimientos nutritivos de los animales por parte de la pradera natural, la que en ciertos períodos no presenta los niveles adecuados de nutrientes para que esto ocurra. Las especies leguminosas pueden ayudar a resolver

$1 \quad$ Universidad de Magallanes, Punta Arenas, Chile. sergio.radic@umag.cl

2 Agri-Food and Biosciences Institute, Newforge Lane, Belfast. United Kingdom. 
esto, porque ellas son la fuente más barata de proteína para los animales sobre estas praderas y son un componente clave para los sistemas de producción. En un experimento de campo en una vega ubicada en la estepa (300-350 mm año-1) de Tierra del Fuego (5309'sur; 6844'oeste), jaulas de exclusión del pastoreo fueron usadas en praderas establecidas (sobre áreas húmedas y secas) para medir el rendimiento y fijación de nitrógeno de Trifolium repens durante la estación de crecimiento. Para medir cuanto nitrógeno ha sido fijado, fue usado el método de la diferencia de nitrógeno. El diseño experimental fue de bloques al azar con tres réplicas. El modelo incluyó área como fuente de variación, analizado por análisis de varianza. El nivel promedio de fijación de nitrógeno fue de $98,7 \mathrm{~kg} \mathrm{~N}$ ha-11y significativamente más $(\mathrm{P} \leq 0,05)$ nitrógeno fue fijado sobre el área húmeda $\left(126 \mathrm{~kg} \mathrm{~N}^{-1}\right)$ que el área seca $\left(71 \mathrm{~kg} \mathrm{~N} \mathrm{ha}^{-1}\right)$.

Palabras clave: Trifolium repens, praderas, Patagonia, humedad de suelo.

\section{INTRODUCCIÓN}

La producción animal en la Región de Magallanes está dada, principalmente, por la disponibilidad de forraje en las praderas naturales (Covacevich \& Ruz 1996), la que en su mayoría no son fertilizadas. Uno de los nutrientes de mayor importancia en los procesos biológicos y que es un componente integral de muchos compuestos en las plantas, es el nitrógeno (N) (Brady \& Weil 2008), el cual, presenta una baja disponibilidad en esta región (Covacevich \& Ruz 1996). Las leguminosas forrajeras pueden ayudar a mejorar esta situación, debido a que presentan una mejor calidad y en algunos casos cantidad de producción que las praderas nativas. Se han introducido diferentes especies para mejorar la producción de los predios, pero las leguminosas son ricas en proteínas y proveen una fuente de nitrógeno derivada de la fijación de nitrógeno del aire (McLaren \& Cameron 1996). La fijación de $\mathrm{N}$ es la habilidad de algunos organismos (bacterias, hongos o actinomycetes) para fijar di-nitrógeno atmosférico (Havlin et al. 2005) y producir compuestos nitrogenados orgánicos (McLaren \& Cameron 1996).

Sin embargo, mediciones de la fijación de nitrógeno en la región no han sido realizadas, siendo importante conocer las cantidades de $\mathrm{N}$ fijadas, las cuales, pueden estimular el crecimiento de las gramíneas. La fijación de $\mathrm{N}$ es también importante si los predios ganaderos fueran manejados orgánicamente, ya que en estos casos, se prohíbe el uso de fertilizantes sintéticos, y las leguminosas serían la principal fuente de $\mathrm{N}$ para estos sistemas. Covacevich (2006), señala que el trébol blanco (Trifolium repens L.) es el pilar de la estrategia de mejoramiento de las praderas del sector húmedo de Magallanes.
Por otra parte, existen factores que limitan la fijación de Ny el crecimiento de los Rhizobios. Ledgard y Steele (1992) y Sprent y Sprent (1990) mencionaron que diferentes factores, como el $\mathrm{N}$ inorgánico del suelo, la acidez, salinidad, deficiencias de nutrientes, toxicidades (aluminio y manganeso), déficit hídrico, altas o bajas temperaturas y enfermedades pueden afectar la fijación biológica de N. Siguiendo la idea anterior, Werner (2007) señaló que la simbiosis puede ser afectada por diferentes factores de stress, como déficit hídrico, deficiencia de los nutrientes del suelo, pH y competición de las especies vegetales vecinas. Finalmente Sanginga et al. (1995) indicó que cuatro factores medio-ambientales, son probablemente los de mayor importancia para la fijación de N: (1) nutrición fosforada, (2) nivel del $\mathrm{N}$ mineral del suelo, (3) déficit hídrico y (4) acidez del suelo.

La técnica de las diferencias de nitrógeno, para medir la fijación de $\mathrm{N}$ es una medición confiable basada en el rendimiento de materia seca (MS) de la planta leguminosa y su contenido de $\mathrm{N}$, además, de la cantidad de $\mathrm{N}$ del suelo en relación a la biomasa total de N. Lo cual, puede ser obtenido cuando una especie usada como control (o referencia) y que no fije $\mathrm{N}$ del aire, está creciendo en el mismo suelo que la especie leguminosa que se desea evaluar (Herridge y Danso 1995; Ledgard \& Steele 1992). Este método puede estimar la fijación de $\mathrm{N}$, bajo el supuesto que la especie leguminosa y la planta control absorben similares cantidades de $\mathrm{N}$ del suelo (Hardarson \& Danso 1993; Herridge \& Danso 1995; Ledgard \& Steele 1992 y Peoples et al. 1989). Por esta razón, que la elección de la planta control es de importancia (Herridge y Danso 1995).

Una de las principales limitaciones de este método es que requiere encontrar una especie no 
fijadora de $\mathrm{N}$ (Peoples et al. 1989) y que no es un método directo (Hardarson \& Danso 1993). Pero las ventajas que posee son que: (i) es un método simple; (ii) se puede incluir las concentraciones de $\mathrm{N}$ del suelo (Peoples et al. 1989) y (iii) se puede obtener una medición de la fijación de $\mathrm{N}$ en un periodo de tiempo determinado (Hardarson y Danso 1993). Boller \& Nosberger (1987); Hardason et al. (1984b); Hoveland \& Richardson (1992); Muller et al. (1993) y Papastylianou (1987) reportaron valores de fijación de $\mathrm{N}$ obtenidos por este método.

El objetivo general de este trabajo fue estimar la cantidad de nitrógeno que es fijado por Trifolium repens en una vega de Tierra del Fuego por la técnica de la diferencia de nitrógeno.

\section{MATERIALES Y MÉTODO}

El experimento fue realizado en la isla de Tierra del Fuego (TF), ubicado en el sector de San Sebastián cercano a los $53^{\circ}$ latitud sur; $68^{\circ}$ longitud oeste. La precipitación del lugar de experimentación varía entre 300 a $350 \mathrm{~mm}_{\text {año }}^{-1}$ y con una temperatura promedio del año en evaluación de $6,7^{\circ} \mathrm{C}$. La especie evaluada fue Trifolium repens L. (trébol blanco) naturalizado.

El muestreo de campo fue realizado con un cuadrante de metal de $0,25 \mathrm{~m}^{2}(50 \times 50 \mathrm{~cm})$ el que fue usado para colectar el material vegetal durante el verano (Frame 1993). El material colectado fue cortado en febrero (150 días posterior al corte inicial y colocación de las jaulas) con una máquina de cortar pasto electrónica (Gardena Accu 6) a una altura de aproximadamente $1 \mathrm{~cm}$ (Laidlaw et al. 1990). La hierba fue guardada en bolsas y pesada fresco. En los laboratorios correspondientes, las muestras de material vegetal fueron lavadas para remover cualquier posible contaminación con suelo, posteriormente fueron secadas a $70^{\circ} \mathrm{C}$ por 48 horas hasta peso constante (Peoples et al. 1989) en un horno de aire circulante. El material vegetal secado fue molido en un molino (marca Brook Crompton Series 2000), tamizado a $1 \mathrm{~mm}$ (MAFF 1986) y almacenado en contenedores hasta realizar los análisis químicos. Para la determinación de la humedad de suelo, muestras de suelo fresco fueron tomadas desde el campo. En el laboratorio, cada muestra de suelo fue dejada sobre una bandeja de aluminio (si la muestra presentaba agregados, estos fueron tamizados), posteriormente se pesó y se secó en un horno a $105^{\circ} \mathrm{C}$ hasta peso constante (alrededor de 16 horas) (Blake y Hartge 1986; Gardner 1986). El contenido de agua fue calculado según la ecuación 1 (Bascomb 1982).

\section{ECUACIÓN 1.}

Contenido de agua (g $\left.100 \mathrm{~g}^{-1}\right)=(($ Masa de muestra tomada - Masa de muestra secada en horno) / Masa de muestra tomada) ${ }^{*} 100$

En el laboratorio del Agri-food Biosciences Institute (AFBI) en Reino Unido fueron realizados los análisis químicos. La determinación del contenido de $\mathrm{N}$ en las muestras de material vegetal fue obtenida después de tamizar la muestra a $0,5 \mathrm{~mm}$, posteriormente $10 \mathrm{mg}$ de material fueron pesados siguiendo la metodología de Laughlin (2006). El contenido de $\mathrm{N}$ de las leguminosas y gramíneas fue determinado por un Analizador de Nitrógeno Carlo Erba NA 1500, unido a un Espectrómetro de Masa VG MM622.

La fijación de nitrógeno fue determinada usando el método de la diferencia de nitrógeno, usando la ecuación 2 (Boddey 1987 \& Peoples et al. 1989). La planta control usada fue el pasto ovillo (Dactylis glomerata L.). Esta planta control se encontraba cerca de las plantas leguminosas usadas, de tal forma de evitar problemas de heterogeneidad (Reichardt et al. 1987). Además, es una de las especies gramíneas naturalizadas más común en la Región de Magallanes.

\section{ECUACIÓN 2.}

$\mathrm{Q}=$ Rendimiento de N (leguminosa) - rendimiento de $\mathrm{N}$ (control)

Rendimiento de $\mathrm{N}=$ (Producción de MS x Porcentaje de N de la especie) / 100.

Donde $\mathrm{Q}$ es la cantidad de nitrógeno de la especie leguminosa derivada de la fijación de $\mathrm{N}_{2}$.

En el experimento conducido se evaluó el efecto de la humedad del suelo (áreas húmeda y seca) sobre el rendimiento de materia seca (MS) y fijación de nitrógeno de Trifolium repens, las jaulas de exclusión del pastoreo (doce en total) fueron co- 
locadas durante la primavera sobre la pradera con trébol y sobre la gramínea control. Tres jaulas sobre trébol y tres en la gramínea en las áreas húmedas y las mismas seis jaulas en las aéreas secas. El diseño experimental fue de bloques al azar con tres réplicas y el modelo incluyó área como fuente de variación. Para las diferencias de humedad de suelo se utilizó un t-test. Para medir la correlación entre los parámetros evaluados se utilizó la correlación de Pearson, usando un valor de significancia para "r" de 0,754 para un $\mathrm{p}$-valor de 0,05 y 0,874 para un p-valor de 0,01.

\section{DISCUSIÓN Y RESULTADOS}

Los contenidos de humedad de suelo promedio del sitio evaluado fueron de $36,1( \pm 3,57) \mathrm{y}$ $25,3( \pm 2,20) \mathrm{g} \mathrm{H}_{2} \mathrm{O} 100 \mathrm{~g}^{-1}$ de suelo húmedo, para áreas húmedas y secas respectivamente. Existiendo diferencias estadísticamente significativas entre áreas. $\mathrm{El} \mathrm{pH}$ al agua $(1: 2,5)$ promedio registrado fue de 6,7 , siendo el $\mathrm{pH}$ para áreas húmedas y secas de 6,5 y 6,9 respectivamente.

La fijación de $\mathrm{N}$ de $\mathrm{TF}$ en la vega fue como promedio $98,7 \mathrm{~kg} \mathrm{~N}^{-1}$. Significativamente $(\mathrm{P} \leq 0,05)$ más $\mathrm{N}$ fue fijado sobre el área húmeda $(126 \mathrm{~kg} \mathrm{~N}$ $\left.\mathrm{ha}^{-1}\right)$ que en la área seca $\left(71 \mathrm{~kg} \mathrm{~N} \mathrm{ha}{ }^{-1}\right)$ de la vega. Diferentes autores (Brock y Kim 1994; Hutchinson et al. 1995; Ledgard y Steele 1992 y Sprent y
Sprent 1990) han mostrado que el trébol blanco es sensible al stress hídrico, lo cual, podría afectar el rendimiento de la materia seca, como también a la fijación de $\mathrm{N}$, lo que respaldaría las diferencias presentadas entre las áreas húmedas y secas. Hubo diferencias significativas en la producción de MS de ambas áreas (fig. 1), presentando una producción de 3.361 y $5.121 \mathrm{~kg} \mathrm{MS} \mathrm{ha}^{-1}$ para las áreas seca y húmeda respectivamente. Covacevich (2006) señala que los mejores resultados de producción de pradera natural con trébol en Magallanes, ha llegado hasta los $8.000 \mathrm{~kg} \mathrm{MS} \mathrm{ha}^{-1}$.

Algunos rangos de fijación de $\mathrm{N}$ por el método de la diferencia de $\mathrm{N}$ en experimentos de campo están entre: 18 - $123 \mathrm{~kg} \mathrm{~N}$ ha $^{-1}$ para Vicia ervilia y Vicia faba respectivamente usando avena como

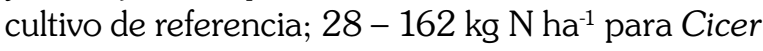
arietinum y Vicia faba usando ballica; 15 - $98 \mathrm{~kg}$ $\mathrm{N}$ ha ${ }^{-1}$ para Cicer arietinum y Vicia ervilia usando cebada (Papastylianou 1987); 9 - $40 \mathrm{~kg} \mathrm{~N} \mathrm{ha}^{-1}$ para diferentes variedades de Glycine max usando a la misma especie pero sin nodulación como referencia y con una dosis de $20 \mathrm{~kg} \mathrm{~N}^{-1}$ (Hardason et al. 1984b); 18 - $140 \mathrm{~kg} \mathrm{~N}^{-1}$ para dos variedades de Lotus corniculatus usando Festuca arundinacea como referencia variando los niveles de fijación de $\mathrm{N}$ dependiendo del año y del lugar evaluado (Hoveland y Richardson 1992); 66 - 299 kg N ha-1 para Trifolium repens en mezcla con Lolium perenne y

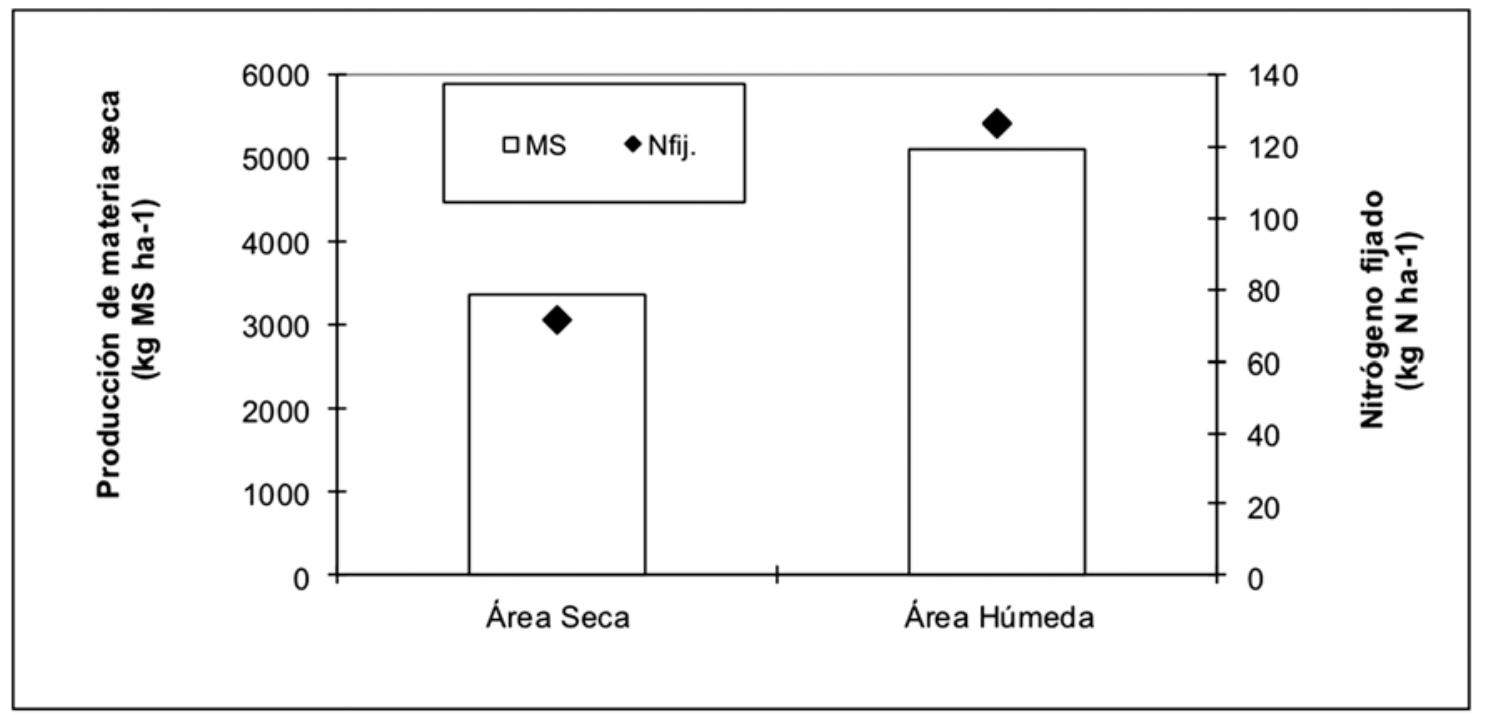

Fig. 1. Gráfica de la producción de materia seca (MS) y la cantidad de nitrógeno fijado (Nfij.) por Trifolium repens en las áreas evaluadas (seca y húmeda) durante 150 días de crecimiento. 
21 - $357 \mathrm{~kg} \mathrm{~N}$ ha ${ }^{-1}$ para Trifolium pratense en mezcla con Lolium perenne (Boller \& Nosberger, 1987).

Otros reportes de niveles de fijación de $\mathrm{N}$ en leguminosas forrajeras por diferentes métodos de medición se encuentran en los rangos; $18-61 \mathrm{~kg} \mathrm{~N}$ ha $^{-1}$ (Farnham \& George, 1994) y $20-90 \mathrm{~kg} \mathrm{~N} \mathrm{ha}^{-1}$ (Heichel et al. 1981) para Lotus corniculatus; para Trifolium repens en mezcla con gramíneas 30 - 52 $\mathrm{kg} \mathrm{N}^{-1}$ (Laidlaw et al. 1990); $20-53 \mathrm{~kg} \mathrm{~N}^{-1}$ (Laidlaw et al. 1996) y $15 \mathrm{~kg} \mathrm{~N}^{-1}$ (Moller Hansen et al. 2002) y para Medicago sativa $68-72 \mathrm{~kg} \mathrm{~N}$ ha $^{-1}$ (West \& Wedin, 1985).

$\mathrm{Si}$ se compara la cantidad promedio de $\mathrm{N}$ fijado por el trébol $(98,7 \mathrm{~kg} \mathrm{~N}$ ha-1) con los fertilizantes salitre $(16 \% \mathrm{~N})$ y urea $(46 \% \mathrm{~N})$, se puede estimar que el aporte anual de $\mathrm{N}$ al sistema podría ser equivalente a $610 \mathrm{~kg}$ de salitre o $212 \mathrm{~kg}$ de urea por hectárea. Covacevich (2006), señala que los aportes por fijación de nitrógeno en la región pueden ser equivalentes a alrededor de $350 \mathrm{~kg}$ de Urea por hectárea al año. Dependiendo de un análisis económico, estos resultados muestran que la inclusión de trébol blanco en sitios aptos para su desarrollo, puede ser un ingreso importante de $\mathrm{N}$ al sistema, además, de ser un aporte nutricional para los ovinos de Tierra del Fuego.

En la figura $2 \mathrm{~A}$, existe una significativa correlación $(r=0,87)$ entre la producción de materia seca y la cantidad de nitrógeno fijado, lo cual es mencionado en otras investigaciones (Carlsson \& Huss-Danell 2003 \& Peoples \& Baldock, 2001). Por lo tanto, cambios en el manejo de la pradera para incrementar la producción de materia seca, podrían aumentar la fijación de N. Peoples y Baldock (2001) mostraron una relación lineal entre producción de materia seca y fijación de N. Las diferencias encontradas en fijación de $\mathrm{N}$, pueden ser debidas, principalmente al contenido de humedad de los sitios. Considerando que el estrés hídrico es uno de los factores que puede afectar la producción de materia seca en trébol, son esperables las diferencias encontradas entre las áreas evaluadas con diferentes contenidos de humedad (área seca y húmeda). Lo anterior, puede ser también uno de los principales factores, por el cual, puede existir variación de la fijación de $\mathrm{N}$ entre años (Ledgard et al. 2001). Ya que las condiciones climáticas (humedad y temperatura) pueden variar y por consiguiente afectar la producción de materia seca de la pradera. En la figura $2 \mathrm{~B}$, se muestra una alta correlación $(\mathrm{r}=0,98)$ entre el $\mathrm{N}$ total de la planta y la fijación de N. Se espera una alta relación entre estos parámetros evaluados, considerando que en promedio el nitrógeno fijado correspondió a un $83 \%$ del $\mathrm{N}$ total, no existiendo diferencias entre áreas y presentando un bajo coeficiente de variación $(<10 \%)$ entre todos los datos usados en la evaluación.

El método de las diferencias de nitrógeno para estimar la fijación de N correlaciona bien con MS, especialmente cuando el $\mathrm{N}$ es el principal nutriente limitante para el crecimiento de la leguminosa en el sistema que se evalúa (Hardason et al. 1984a). Sáez (1995) señala que en la mayoría de los suelos muestreados en la región de Magallanes los niveles de disponibilidad de nitrógeno serían insuficientes para satisfacer los requerimientos de los cultivos. La
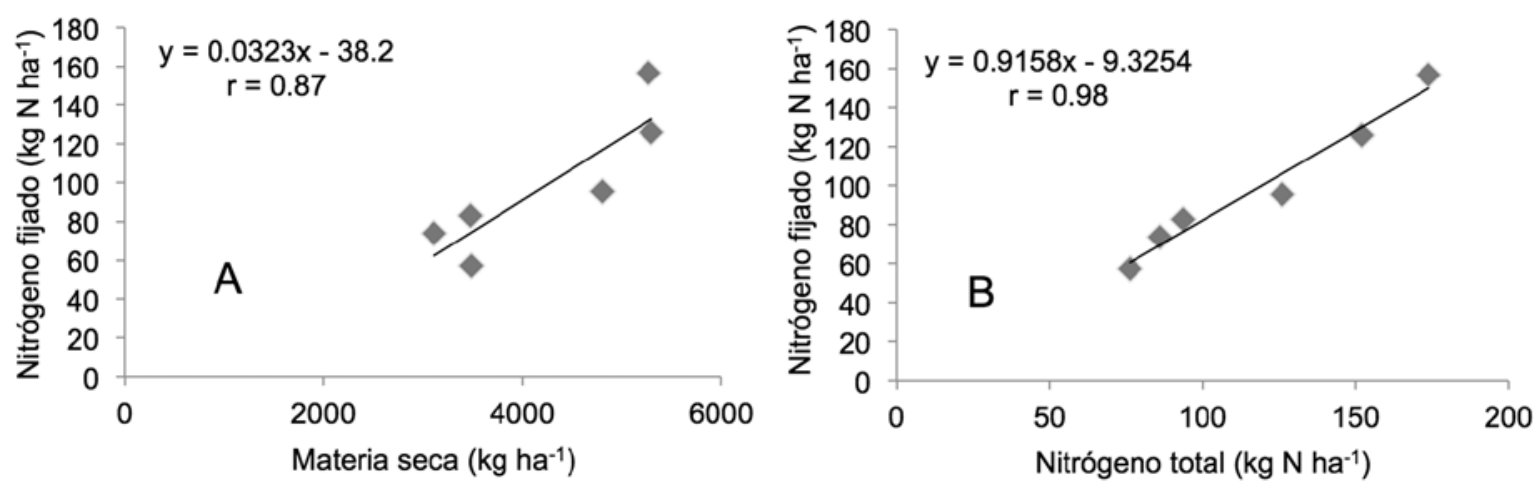

Fig. 2. (A) Correlación entre producción de materia seca y cantidad de nitrógeno fijado. (B) Correlación entre nitrógeno total en la planta y cantidad de nitrógeno fijado. 
metodología usada presenta ventajas y desventajas para determinar la fijación de $\mathrm{N}$, como fue mencionado previamente, pero al no existir investigaciones previas sobre la estimación de la fijación de $\mathrm{N}$ en la región de Magallanes, el método de las diferencias de $\mathrm{N}$ resulta útil para tener una primera aproximación de las cantidades de $\mathrm{N}$ fijadas en la región. La etapa siguiente es determinar la fijación de $\mathrm{N}$ por algún método más directo, como la metodología de ${ }^{15} \mathrm{~N}$, la que es avalada por varios autores (Danso 1995; Herridge \& Danso, 1995; Ledgard \& Steele, 1992). Lo anterior, no invalida los resultados encontrados por el método de las diferencias de $\mathrm{N}$, ya que se han encontrado investigaciones de campo (Shock 1984) donde este método fue altamente correlacionado con la técnica de ${ }^{15} \mathrm{~N}$, cuando fue usado para Trifolium subterraneum.

Debido al clima adverso en general, y a las bajas temperaturas en particular, se señala que la fijación de $\mathrm{N}$ en ecosistemas de alta latitud es baja. Cralle \& Heichel (1982) y Nesheim \& Boller (1991), encontraron que la temperatura puede ser una importante limitación para la fijación de $\mathrm{N}$ en diferentes leguminosas. Carlsson \& Huss-Danell (2003) también mencionaron que en ambientes de baja temperatura, efectos de gran escala por localización geográfica son menores comparados con los efectos locales como tipo de suelo, microclima y manejo. Por su parte, Hoveland \& Richardson (1992) encontraron que la fijación de $\mathrm{N}$ varió entre lugares de diferente altitud, entre cultivares de una misma especie y entre años de evaluación. Por otro lado, se debe evitar un exceso de material vegetal en la pradera, que pueda producir sombra al trébol. Investigaciones (Schwank et al. 1986) han demostrado que la competencia por luz es uno de los principales factores que limita la proporción de trébol blanco en las praderas. Además, los cambios en el rendimiento del $\mathrm{N}$ obtenido por fijación están relacionados a la producción de MS del trébol, por lo que cualquier manejo que mejore la producción de MS incrementaría la fijación simbiótica de nitrógeno.

Esta investigación mostró que la fijación de $\mathrm{N}$ en la especie Trifolium repens es posible en sectores de baja precipitación y temperatura. Además, esta especie aporta $\mathrm{N}$ para los sistemas de producción ovina extensivos, donde habitualmente no se realiza fertilización de las praderas y puede transferir parte de este $\mathrm{N}$ a la gramínea acompañante, como ha sido encontrado en algunas investigaciones (Boller y Nosberger 1987 y Broadbent et al. 1982).

\section{AGRADECIMIENTOS}

El trabajo presentado en esta publicación fue parte de una tesis doctoral de S. Radic, desarrollada en Queen's University Belfast (Reino Unido), con el apoyo de una beca MECESUP, el respaldo de la Universidad de Magallanes y del Agri-Food Bioscience Institute (Reino Unido).

\section{LITERATURA CITADA}

Bascomb, C.L. 1982. Physical and chemical analyses of $<2$ mm samples. p. 14-41. In. Avery, B.W. and Bascomb C.L. (eds.) Soil survey laboratory Methods. Soil survey technical monograph No. 6. Harpenden, Herts. United Kingdom.

Blake, G.R. \& K.H. Hartge 1986. Bulk density. p. 363-375. In Klute, A. (ed.), Methods of soil analysis. Part 1. Physical and mineralogical methods. $2^{\text {nd }}$ Ed. Madison, Wisconsin, USA.

Boller, B.C. \& J. Nosberger 1987. Symbiotically fixed nitrogen from field-grown white and red clover mixed with ryegrasses at low levels of ${ }^{15} \mathrm{~N}-$ fertilization. Plant and Soil, 104: 219-226.

Boddey, R.M. 1987. Methods for quantification of nitrogen fixation associated with gramineae. Critical Review Plant Science, 6: 209-266.

Brady, N. \& R. Weil 2008. The Nature and Properties of soils. Fourteenth edition. Prentice Hall, New Jersey, USA. 975 p.

Broadbent, F.E., T. Nakashima \& G.Y. Chang 1982. Estimation of nitrogen-fixation by isotopedilution in field and greenhouse experiments. Agronomy Journal, 74(4): 625-628.

Brock, J.L. \& M.C. Kim 1994. Influence of the stolon/ soil surface interface and plant morphology on the survival of white clover during severe drought. Proceedings of the New Zealand Grassland Association, 56: 187-191.

Carlsson, G. \& K. Huss-Danell 2003. Nitrogen fixation in perennial forage legumes in the field. Plant and Soil, 253: 353-372.

Covacevich, N. \& E. Ruz 1996. Praderas en la zona austral: XII Región (Magallanes). En: Ruiz, I (Ed.) Capitulo 37, Praderas para Chile, 2a Edición. INIA, MINAGRI, Chile. 639-655 pp.. 
Covacevich, N. 2006. Trébol para Magallanes. Edición Especial, Tierra Adentro. Instituto de Investigación Agropecuaria. Punta Arenas, Chile. 28-31 pp.

Cralle, H.T. \& G.H. Heichel 1982. Temperature and chilling sensitivity of nodule nitrogenase activity of unhardened alfalfa. Crop Science, 22: 300-304.

Danso, S.K.A. 1995. Assessment of biological nitrogen fixation. Plant and Soil, 42: 33-41.

Farnham, D.E. \& R.J. George 1994. Dinitrogen fixation and nitrogen transfer in Birdsfoot trefoil-Orchardgrass communities. Agronomy Journal, 86: 690-694.

Frame, J. 1993. Herbage mass. pp. 39-67. In Davis, Baker, Grant and Laidlaw (eds.). Sward measurement handbook. $2^{\underline{a}}$ ed. British Grassland Society, Reading, United Kingdom

Gardner, W.H. 1986. Water content. pp. 493-544. Klute, A. (ed.), In Methods of soil analysis. Part 1. physical and mineralogical methods. $2^{\text {nd }}$ Ed., Madison, Wisconsin, USA.

Hardason, G. \& S.K.A. Danso 1993. Methods for measuring biological nitrogen fixation in grain legumes. Plant and Soil, 152: 19-23.

Hardason, G., F. Zapata \& S.K.A. Danso 1984a. Field evaluation of symbiotic nitrogen fixation by rhizobial strains using ${ }^{15} \mathrm{~N}$ methodology. Plant and Soil, 82: 369-375.

Hardason, G., F. Zapata \& S.K.A. Danso 1984 b. Effect of plant genotype and nitrogen fertilizer on symbiotic nitrogen fixation by soybean cultivars. Plant and Soil, 82: 397-405.

Havlin, J.L., I.D. Beaton, S.L Tisdale \& W.L. Nelson 2005. Soil Fertility and Fertilizers. $7^{\text {th }}$ edition. Prentice Hall, New Jersey, USA. $515 \mathrm{p}$.

Heichel, G.H., C.P. Vance \& D.K. Barnes 1981. Symbiotic nitrogen fixation of alfalfa, birdsfoot trefoil and red clover. pp. 336-338. In. Smith, A. and Hays, V. (eds.) Proceeding of the XIV International Grassland Congress, Lexington, Kentucky, USA.

Herridge, D.F. \& S.K.A. Danso 1995. Enhancing crop legume $\mathrm{N}_{2}$ fixation through selection and breeding. Plant and Soil, 174: 51-82.

Hoveland, C.S. \& M.D. Richardson 1992. Nitrogen fertilization of tall fescue-birdsfoot trefoil mixtures. Agronomy Journal, 84: 621-627.
Hutchinson, K.J., K.L. King \& D.R. Wilkinson 1995. Effects of rainfall, moisture stress, and stocking rate on persistence of white clover over 30 years. Australian Journal of Experimental Agriculture, 35(7): 1039-1047.

Laidlaw, A. S., Christie, P. y H. W. Lee 1996. Effect of white clover cultivar on apparent transfer of nitrogen from clover to grass and estimation of relative turnover rates of nitrogen in roots. Plant and Soil, 179: 243-253.

Laidlaw, A. S., P. Christie \& A.S. Withers 1990. Comparison between isotope dilution and acetylene reduction methods to estimate $\mathrm{N}_{2}$ fixation rate of white clover in grass/clover swards. Grass and Forage Science, 45: 295-301.

Laughlin, R. J. 2006. Operation of a Carlo Erba elemental analyser interfaced to a europa scientific tracermass for measuring the concentration and enrichment of nitrogen and carbon in plant material, soils and liquids. Internal Circulation, Agri-Food Biosciences Institute, Northern Ireland, United Kingdom. $80 \mathrm{pp}$.

Ledgard, S., M.S. Sprosen, J.W. Penno \& G.S. Rajendram 2001. Nitrogen cycling in low input legume-based agriculture, with emphasis on legume/grass pastures. Plant and Soil, 229: 177-187.

Ledgard, S. \& K. Steele 1992. Biological nitrogen fixation in mixed legume/grass pastures. Plant and Soil, 141: 137-153.

McLaren, R.G. \& K.C. Cameron 1996. Soil science, sustainable production and environmental protection. $2^{\text {nd }}$ ed. Oxford University Press. Auckland, New Zealand. 304 p.

Ministry of Agriculture, Fisheries and Food (MAFF). 1986. The analysis of agricultural materials: a manual of the analytical methods used by the Agricultural Development and Advisory Service. $3^{\text {rd }}$ ed. HMSO, London, United Kingdom. 248 p.

Moller Hansen, E., H. Hogh-Jensen \& J. Djurhuus 2002. Biological nitrogen fixation in a grazed perennial grass/clover ley and correlation with herbage and soil variables. European Journal of Agronomy, 16: 309-320.

Muller, S., P.A.A. Pereira \& P. Martin 1993. Effect of different levels of mineral nitrogen on 
nodulation and $\mathrm{N}_{2}$ fixation of two cultivars of common bean (Phaseolus vulgaris L.). Plant and Soil, 152: 139-143.

Nesheim, L. \& B.C. Boller 1991. Nitrogen fixation by white clover when competing with grasses at moderately low temperatures. Plant and Soil, 133: 47-56

Papastylianou, I. 1987. Amount of nitrogen fixed by forage, pasture and grain legumes in Cyprus, estimated by the A-value and a modified difference method. Plant and Soil, 104: 23- 29.

Peoples, M.B. \& J.A. Baldock 2001. Nitrogen dynamics of pastures: nitrogen fixation inputs, the impact of legumes on soil nitrogen fertility, and the contributions of fixed nitrogen to Australian farming systems. Australian Journal of Experimental Agriculture, 41: 327-346.

Peoples, M.B., A.W. Faizah, B. Rerkasem \& D.F. Herridge 1989. Methods for evaluating nitrogen fixation by nodulated legumes in the field. ACIAR, Monograph No. 11. Canberra, Australia. 76 p.

Reichardt, K., G. Hardarson, F. Zapata, C. Kirda \& K.A. Danso 1987. Site variability effect on field measurement of symbiotic nitrogen fixation using the ${ }^{15} \mathrm{~N}$ isotope dilution method. Soil Biology and Biochemistry, 19(4): 405-409.
Saez, C. 1995. Fertilidad de los Suelos de la Región de Magallanes. Universidad de Magallanes, Chile. 32 pp.

Sanginga, N., Vanlauwe, B. y Danso, S.K.A. 1995. Management of biological $\mathrm{N}_{2}$ fixation in alley cropping systems: estimation and contribution to N balance. Plant and Soil, 174: 119-141.

Schwank, O., H. Blum \& J. Nosberger 1986. The influence of irradiance distribution on the growth of white clover (Trifolium repens L.) in differently managed canopies of permanent grassland. Annals of Botany, 57: 273-281.

Shock, C.C. 1984. Nitrogen fixation by subclover associations fertilized with sulphur. Plant and Soil, 81: 323-332.

Sprent, J.I. \& P. Sprent 1990. Nitrogen fixing organisms. Chapman and Hall. University Press, Cambridge. United Kingdom. 256 pp.

Werner, D. 2007. Molecular biology and ecology of the Rhizobia-legume symbiosis. pp. 237-266. In. Pinton, R., Varanini, Z. and Nannipieri, P. (eds.) The rhizosphere. Biochemistry and organic substances at the soil-plant interface. Second edition. CRC Press. Taylor \& Francis Group. USA.

West, C.P. \& W.F. Wedin 1985. Dinitrogen fixation in Alfalfa-Orchardgrass pastures. Agronomy Journal, 77: 89-94. 